\title{
Detection of a fluorescent-labeled avidin-nucleic acid nanoassembly by confocal laser endomicroscopy in the microvasculature of chronically inflamed intestinal mucosa
}

\author{
This article was published in the following Dove Press journal: \\ International Journal of Nanomedicine \\ 8 January 2015 \\ Number of times this article has been viewed
}

\begin{abstract}
Andrea Buda',*
Sonia Facchin ${ }^{1, *}$

Elisa Dassie ${ }^{2}$

Elisabetta Casarin ${ }^{3}$

Mark A Jepson ${ }^{4}$

Helmut Neumann ${ }^{5}$

Giorgia Hatem'

Stefano Realdon ${ }^{6}$

Renata D'Incà'

Giacomo Carlo Sturniolo'

Margherita Morpurgo 3

'Department of Surgical, Oncological, and Gastroenterological Sciences, University of Padova, ${ }^{2}$ Department of Molecular Medicine, University of Padova, Padova, Italy; ${ }^{3}$ Department of Pharmaceutical and Pharmacological Sciences, University of Padova, Padova, Italy; ${ }^{4}$ School of Biochemistry and Wolfson Bioimaging Facility, University of Bristol, Bristol, UK; ${ }^{5}$ Ludwig Demlig Endoscopic Center of Excellence, ESGE Endoscopy Training Center, University of Erlangen-Nuremberg, Erlangen, Germany; ${ }^{6}$ Veneto Institute of Oncology IOV-IRCCS, Padova, Italy

*These authors contributed equally to this work
\end{abstract}

Correspondence: Margherita Morpurgo Pharmaceutical and Pharmacological Sciences Department, University of Padova, via Marzolo 5, 35I3 I

Padova, Italy

Tel +390498275330

Fax +390498275366

Email margherita.morpurgo@unipd.it

\begin{abstract}
Inflammatory bowel diseases are chronic gastrointestinal pathologies causing great discomfort in both children and adults. The pathogenesis of inflammatory bowel diseases is not yet fully understood and their diagnosis and treatment are often challenging. Nanoparticle-based strategies have been tested in local drug delivery to the inflamed colon. Here, we have investigated the use of the novel avidin-nucleic acid nanoassembly (ANANAS) platform as a potential diagnostic carrier in an experimental model of inflammatory bowel diseases. Fluorescentlabeled ANANAS nanoparticles were administered to mice with chemically induced chronic inflammation of the large intestine. Localization of mucosal nanoparticles was assessed in vivo by dual-band confocal laser endomicroscopy. This technique enables characterization of the mucosal microvasculature and crypt architecture at subcellular resolution. Intravascular nanoparticle distribution was observed in the inflamed mucosa but not in healthy controls, demonstrating the utility of the combination of ANANAS and confocal laser endomicroscopy for highlighting intestinal inflammatory conditions. The specific localization of ANANAS in inflamed tissues supports the potential of this platform as a targeted carrier for bioactive moieties in the treatment of inflammatory bowel disease.
\end{abstract}

Keywords: confocal laser endomicroscopy, inflammatory bowel disease, diagnostics, dextran sodium sulfate, avidin-nucleic acid nanoassembly, fluorescent nanoparticles, ulcerative colitis

\section{Introduction}

Crohn's disease and ulcerative colitis are chronic inflammatory bowel diseases (IBD) with a severe impact on social life, reducing quality of life and the ability to work as a consequence of increasing disability. IBD are chronic diseases with symptoms of bloody diarrhea and abdominal cramp. They generally begin in young adulthood and last throughout life, with nearly $80 \%$ of patients with Crohn's disease and $30 \%$ of those with ulcerative colitis requiring intestinal surgery. There is a strong need for novel strategies to improve the diagnosis and treatment of IBD. Indeed, the pathogenesis of these diseases is not yet fully understood ${ }^{1-3}$ and their diagnosis and treatment are often challenging. Standard treatments to induce disease remission include aminosalicylates, corticosteroids, and immunosuppressive agents such as thiopurines and anti-tumor necrosis factor- $\alpha$. Immunomodulator and combination therapies constitute a significant advance in the management of IBD. ${ }^{4-7}$ However, the onset of action is delayed with some of these drugs, and/or important adverse systemic effects often 
occur, including anaphylactic/anaphylactoid ${ }^{8,9}$ and lupus symptoms, and bacterial, viral, or fungal infections. ${ }^{10-12}$

A possible way to reduce side effects without losing efficacy is to use targeting strategies to increase drug tropism to the inflamed sites. This strategy could improve drug therapeutic indices and the development of more effective treatment schedules. In recent years, several nanoparticle and colloidal systems have shown enhanced accumulation at inflamed sites due to hypervascularization ${ }^{13}$ or in tumors because of ineffective lymphatic drainage. ${ }^{14}$ These systems have the advantage of being able to carry a high drug payload/ circulating unit, and in principle they could address the need for targeted delivery of active agents to IBD sites. Further, the mucosal epithelium is characterized by leaky structure in $\mathrm{IBD}^{15}$ and this particular feature may offer an opportunity for selective drug targeting through use of colloidal carriers.

In this work, we evaluated the behavior of a recently described nanoparticle platform, ie, an avidin-nucleic acid nanoassembly (ANANAS), when administered parenterally in a mouse model of IBD. ANANAS are fully biodegradable and biocompatible particles consisting of a compositionally defined poly-avidin core capable of accommodating more than 1,000 biotin-linked moieties of different kinds with unique stoichiometric and compositional control through a one-pot synthetic process. ${ }^{16}$ Recently, the potential of the ANANAS formulation as a theranostic carrier for intravenous administration was demonstrated. The nanoparticles are capable of circulating for at least 2 hours before being eliminated within the next 2 days through classic scavenging systems with no apparent toxicity and low immunogenicity. ${ }^{17}$ These findings paved the way for further exploitation of this nanoparticle tool for in vivo diagnostics and delivery applications. ${ }^{18}$ The ANANAS platform is especially interesting because it also meets the current need for convenient and reproducible preparation methods for multifunctional nanocarriers. ${ }^{19}$ Multifunctionality in nanoparticles, while offering great potential for targeted treatments, significantly increases the complexity of preparation methods. As a consequence, the availability of standardized and reproducible synthetic procedures to control the multifunctional carrier composition is mandatory for translating research findings into clinical practice.

Here, in a further attempt to evaluate the clinical potential of this nanoparticle technology in nanomedicine, we generated a near red-emitting fluorophore-labeled ANANAS formulation (ANANAS-red ${ }^{681}$ ), and performed a preliminary investigation of its distribution after intravenous administration in the colonic mucosa of an experimental model of
IBD. Confocal laser endomicroscopy (CLE) is a noninvasive fluorescence-based diagnostic tool for assessment of in vivo histology of the mucosa at subcellular resolution during ongoing endoscopy. ${ }^{20}$ Probe-based CLE is a system designed for the in vivo characterization of the gastrointestinal mucosa and is currently used in the preclinical ${ }^{21,22}$ and clinical diagnostics ${ }^{23-25}$ of IBD. Intravenous administration of the green fluorescent dye fluorescein as a contrast agent allows visualization of the morphology of mucosal crypts and microvasculature. Dual-band CLE is a research-dedicated system that generates two-color laser excitation $(488 \mathrm{~nm} / 660 \mathrm{~nm})$ and permits real-time dual-band fluorescence detection (500-630 nm/680-800 nm). This tool allows detection of two different fluorophore-related probes within the mucosal environment of interest and thus simultaneous visualization of different structures and/or targets.

In this pilot study, ANANAS-red ${ }^{681}$ was administered intravenously together with low molecular weight fluorescein solution in order to determine the distribution of nanoparticles within the colonic mucosa, the architecture of which is highlighted by fluorescein. The red ${ }^{681}$ fluorophore was used to enable nanoparticle visualization in the colonic microvasculature by CLE. In principle, the fluorophore can be substituted with bioactive compounds to develop a therapeutic application.

\section{Materials and methods Animal study approval}

Procedures involving animals and their care were conducted in compliance with Research Project 27/2013 (protocol number 28893 May 13, 2013). The project proposal was approved by the Institutional Animal Care and Use Committee of the University of Padova according to Italian and European Commission regulations on Laboratory Animal Welfare and Protection (legislative decree number 116/92), which acknowledges the European Directive 86/609 EU.

\section{Induction of dextran sodium sulfate colitis}

Chronic colitis was induced in Balb/c mice ${ }^{26,27}$ (female, aged 8-10 weeks) with dextran sodium sulfate (DSS, molecular mass $40,000 \mathrm{Da}$ ), according to a protocol specifically optimized and described in detail in the Supplementary material. During treatment, animal body weight, rectal bleeding, stool consistency, and the presence of blood in stools were assessed daily. Assessment of chronic inflammation was confirmed by histological analysis. ${ }^{28}$ Mice given untreated drinking water were used as controls. 


\section{Synthesis of biotin derivatives used for nanoparticle assembly}

The synthetic steps are summarized in Figure 1. Compound 1 and biotin- $\mathrm{C}_{6}-\mathrm{NH}_{2}$ (compound 2) were obtained as described elsewhere. ${ }^{29}$ Compound 3 was obtained by reacting compound 2 with fluorescent red $^{681}$ succinimidyl ester ( red $^{681}$-NHS, Sigma Aldrich, St Louis, MO, USA, catalog number 53433) in dry dimethyl sulfoxide at a molar ratio of $1: 1.1$ in the presence of triethylamine.

In more detail, $45.9 \mu \mathrm{L}$ of a biotin- $\mathrm{C}_{6}-\mathrm{NH}_{2} \cdot \mathrm{HCl}$ solution in dry dimethyl sulfoxide $(9.3 \mathrm{mg} / \mathrm{mL})$ was added to 49 $\mu \mathrm{L}$ of red ${ }^{681}$-NHS in dry dimethyl sulfoxide $(20 \mathrm{mg} / \mathrm{mL})$, followed twice by $7.7 \mu \mathrm{L}(2 \times 7.7)$ of triethylamine $1: 50$ in dry dimethyl sulfoxide. The product (biotin-red ${ }^{681}$ ) was characterized for its ability to bind avidin through gel permeation chromatography and for its fluorescence intensity before and after avidin binding (Supplementary material).

\section{Preparation of ANANAS-red ${ }^{681}$}

The term ANANAS refers to a series of poly-avidin nanoparticle formulations originating from the high affinity interaction of avidin with nucleic acids. ${ }^{30}$ These buffersoluble nanoparticles are formed by a spontaneous double self-assembly in which a nucleic acid molecule acts as a central element around which several avidins (one for each 14 base pairs) nucleate, leading to toroidal assemblies (diameter approximately $120 \mathrm{~nm}$ ). In a second self-assembly process, a defined amount of biotinylated poly(ethylene glycol) (PEG) is added to permit buffer solubility and increase stability. ${ }^{29}$ Finally, the ANANAS assemblies are polymerized forms of avidin. In this study, red ${ }^{681}$-labeled ANANAS nanoparticles were synthesized in collaboration with ANANAS Nanotech (Padova, Italy) according to a procedure described elsewhere. ${ }^{17}$ In detail, a highly PEGylated ANANAS nanoparticle formulation was prepared and stabilized by mixing plasmid DNA and a complex of avidin and compound 1 at 1:0.25 molar ratio. ${ }^{17,29}$ After purification and ultravioletvisible characterization of the nanoassembly, biotin- $\mathrm{C}_{6}-\mathrm{red}^{681}$ (compound 3) was added to at 1:3 avidin:biotin molar ratio. Finally, the mixture was purified from low molecular weight derivatives by gel permeation chromatography using a Sepharose 6-FF column on a fast protein liquid chromatography system (AKTA purifier, GE Healthcare, Little Chalfont, UK) with $10 \mathrm{mM}$ phosphate, $150 \mathrm{mM} \mathrm{NaCl} \mathrm{pH} 7.4$ (phosphatebuffered saline) as the eluent. Four hundred and seventy five fluorophores/nanoparticles were found to be stably attached, as determined from the assembly ultraviolet-visible spectrum on the basis of the $280 \mathrm{~nm}$ (avidin, DNA, and red ${ }^{681}$ ) and $681 \mathrm{~nm}\left(\right.$ red $^{681}$ ) absorption values. The assembly size

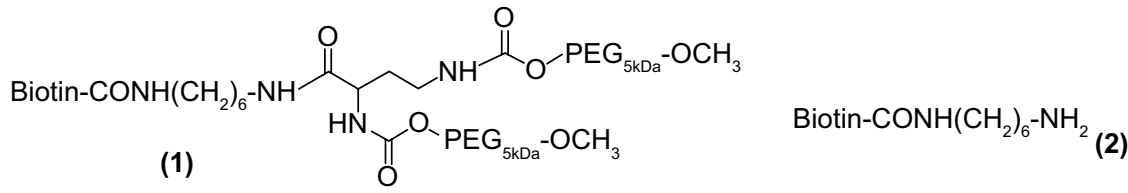

Biotin-(mPEG $\left.{ }_{5 k D a}\right)_{2}$

Biotin- $\mathrm{C}_{6}-\mathrm{NH}_{2}$

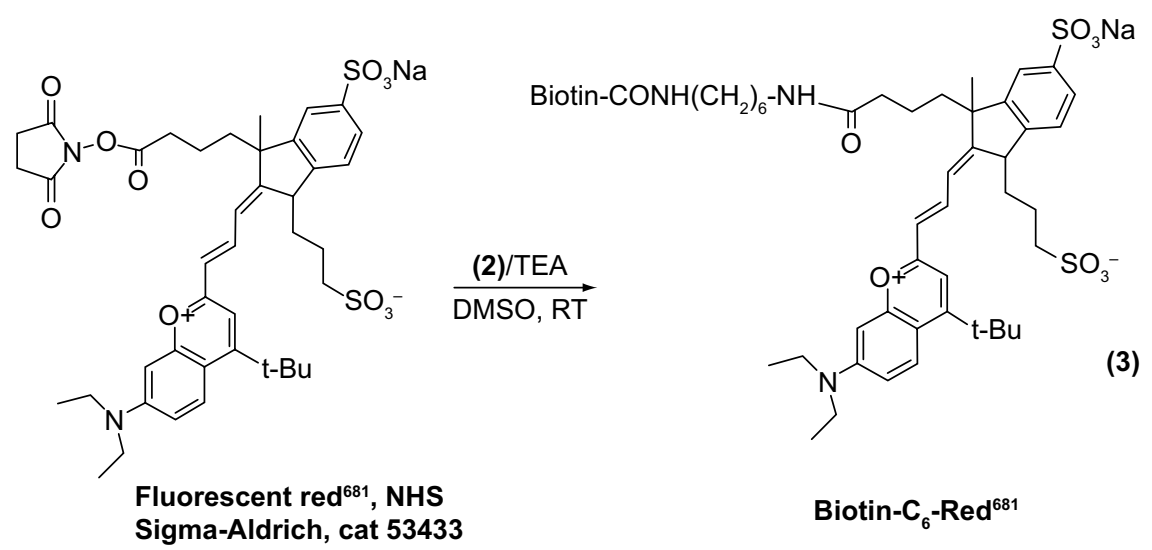

Figure I Synthesis of biotin derivatives used in assembly of ANANAS-red ${ }^{681}$

Abbreviations: ANANAS, avidin-nucleic acid nanoassembly; DMSO, dimethyl sulfoxide; TEA, triethylamine; RT, room temperature; NHS, N-hydroxysuccinimide; PEG: poly(ethylene glycol). 
was approximately $120 \mathrm{~nm}$ as measured by dynamic light scattering (Supplementary material).

\section{Confocal laser endomicroscopy}

We used the Cellvizio Dual Band system integrated with Proflex Ultra Mini O/Z probes (Mauna Kea Technologies, Paris, France) and a flexible endoscope (MAS-GM, Olympus, Italia S.r.1., Milano, Italy). Post-processing imaging analysis was performed using IC-viewer software. CLE was carried out on anesthetized healthy and diseased mice after endoscopic confirmation of mucosal damage. Fluorescein in phosphatebuffered saline solution, in the presence or absence of ANANAS-red ${ }^{681}$ (100 $\left.\mu \mathrm{g} / \mathrm{mouse}\right)$, was administered intravenously $(100 \mu \mathrm{L})$ in healthy and DSS-treated animals (three animals per condition) and CLE images were acquired 15-30 minutes after injection. Longer observation times could not be used due to the rapid fluorescein clearance.

\section{Results and discussion Optimization of fluorescein dosage for dual-band CLE investigation}

Preliminary experiments were carried out to optimize experimental conditions to provide the most valuable biological information using the dual-band probe. When using dual-band fluorescence imaging, if the signal generated by one fluorophore is too high, signal cross-over into the second channel may occur, resulting in false-positive readings. In our experiments, the concentration of red ${ }^{681}$ dye in the injected nanoparticle formulation was several orders of magnitude lower $(0.00167 \% \mathrm{w} / \mathrm{v})$ than that of the fluorescein dye normally used for single-band CLE-assisted visualization of the microvasculature (between $10 \%$ and $0.5 \% \mathrm{w} / \mathrm{v}) .{ }^{31}$ Since this was the first application of dual-band CLE for preclinical research in IBD, we tested a range of fluorescein concentrations to eliminate the risk of a falsepositive signal from fluorescein interfering with specific red $^{681}$-related signals.

In this preliminary investigation, healthy and inflamed animals were injected with different fluorescein solutions $(0.1 \%, 1.0 \%$, and $10 \% \mathrm{w} / \mathrm{v}$ in phosphate-buffered saline) at $100 \mu \mathrm{L} /$ dose to identify the fluorophore concentration allowing the morphology of the vasculature (in the green channel) to be reliably distinguished without generating cross-over of fluorescent emission in the red channel. Only the intermediate fluorescein concentration tested was found to be suitable for our purposes (Figure 2).

Administration of the $10 \%$ dye solution generated a strong and diffuse signal in the green channel, together with a significant bleed-through of fluorescent emission in the red channel. The diffuse signal, likely due to diffusion of the dye from the vessels to the surrounding stroma, impaired visualization of crypt and microvasculature architecture. In addition, the strong spectral cross-over effect prevented reliable identification of a positive signal due to the red-emitting dye in the red channel. Using $0.1 \%$ fluorescein, no bleedthrough was observed in either healthy or inflamed mucosae, but the microvasculature pattern was poorly defined. Finally, $1 \%$ fluorescein was found to be suitable for our purposes, because it allowed the crypt and microvascular architecture to be visualized in a manner similar to that possible with use of $10 \%$ fluorescein in human clinical practice, ${ }^{23}$ with negligible spectral cross-over in either healthy or chronically inflamed mucosae.

\section{ANANAS-red ${ }^{681}$ reaches mucosal microvasculature in inflamed but not healthy bowel}

ANANAS-red ${ }^{681}$ were coinjected with $1.0 \%$ fluorescein. Selective detection of nanoparticles was observed exclusively in the inflamed tissue (Figure 3). The red signal appears in the inflamed vessels (highlighted by the green fluorescein) only, and is clearly related to the presence of ANANAS-red ${ }^{681}$, as confirmed by the merged images. The speckled red signal is likely due to the low fluorophore concentration $(0.00167 \%$ $\operatorname{red}^{681}$ versus $1 \%$ fluorescein $\mathrm{w} / \mathrm{v}$ ), the discrete concentration of nanoparticles, and the lower relative brightness of $\mathrm{red}^{681}$ as compared with fluorescein (not shown). The results therefore confirm that, like other colloidal drug carriers, ANANAS nanoparticles are capable of selectively reaching inflamed tissues. Of note, when CLE probing was performed on healthy regions of the patchily inflamed colon, no red $^{681}$ signal was observed (not shown). This indicates that the system can discriminate between healthy and inflamed areas in the same organ.

\section{Conclusion}

In these preliminary studies, dual-band CLE was used for the first time to characterize the architecture of the microvasculature and visualize fluorescent-labeled nanoparticles simultaneously in an in vivo model. A distinctive pattern of distribution of intravascular nanoparticles was observed in chronic inflamed mucosa compared with healthy controls. The results confirm that ANANAS nanoparticle-based delivery strategies have the ability to reach the microvasculature of inflamed bowel mucosa together with their cargo. This result further supports the potential of this novel soft biodegradable 


\section{Fluorescein}

$10 \%$

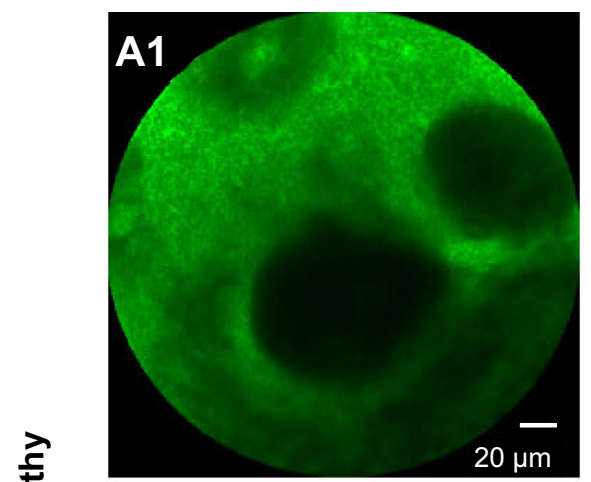

离
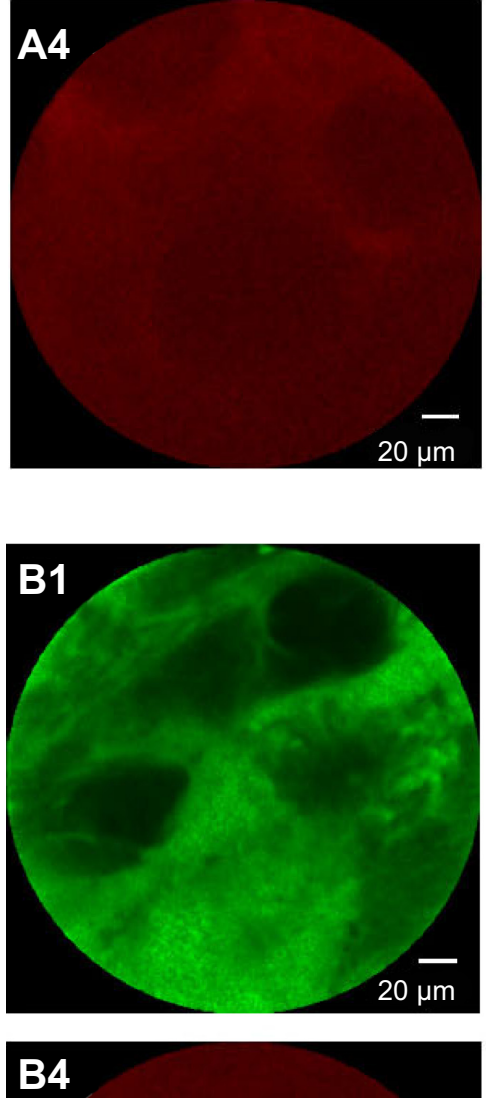

$20 \mu \mathrm{m}$
$1 \%$
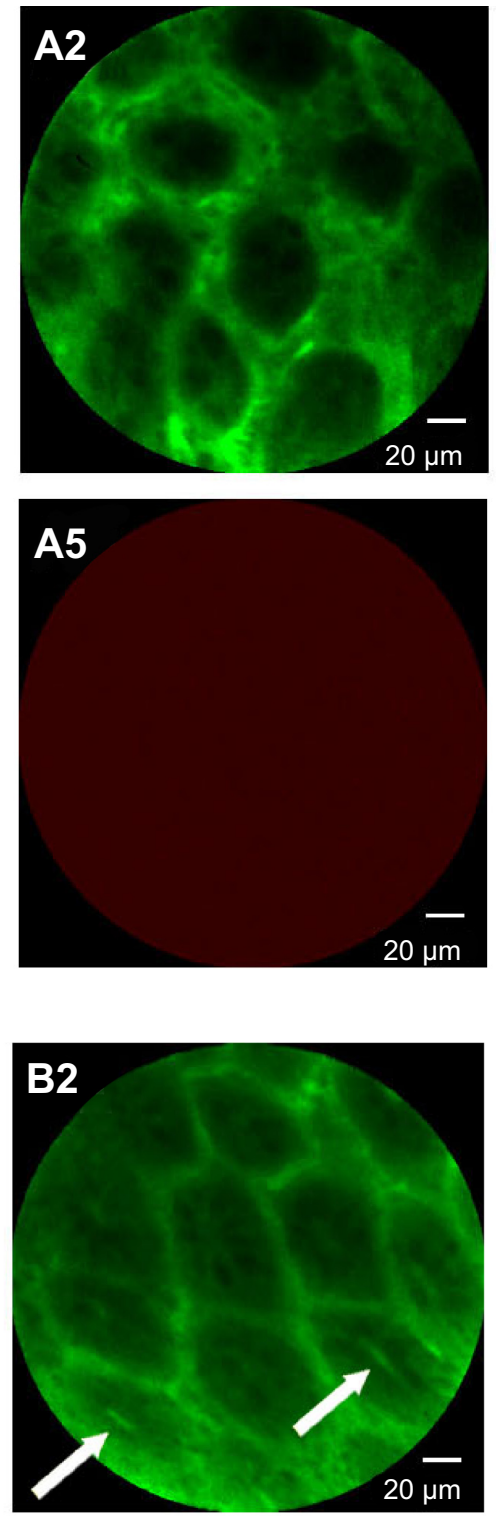

B5

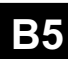

$20 \mu \mathrm{m}$
$0.1 \%$
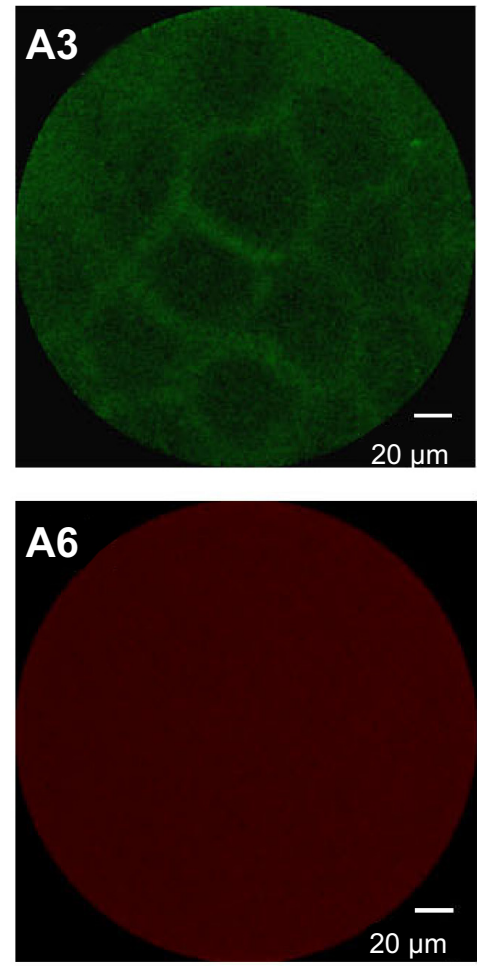

\section{B3}

$20 \mu \mathrm{m}$

\section{B6}

$20 \mu \mathrm{m}$

Figure 2 Confocal laser endomicroscopic images of healthy and chronically inflamed mucosa in the green and red channels.

Notes: The images were obtained after intravenous administration of fluorescein at different concentrations ( $10 \%$, I. $0 \%$, and $0.1 \%$ w/v). Each image represents one example of three per group of animals per condition. The images obtained in the green channel (AI-A 3 and $\mathbf{B} \mathbf{I}-\mathbf{B} \mathbf{3})$ show the hexagonal honeycomb appearance of the mucosa. Nonideal fluorescein concentrations yield either overexposed images ( $10 \%$, A I and B I) or loss of details $(0.1 \%$, A3 and B3). Normal mucosa shows a network of capillaries surrounding the crypt openings with a honeycomb appearance. In inflamed mucosa, the crypt lumen is large and variably shaped, with fluorescein leakage into the crypt openings (arrows in B2). In the red channel (A4-A6 and B4-B6), any visible structure (A4 and B4) is due to fluorescein-related signal cross-over. 


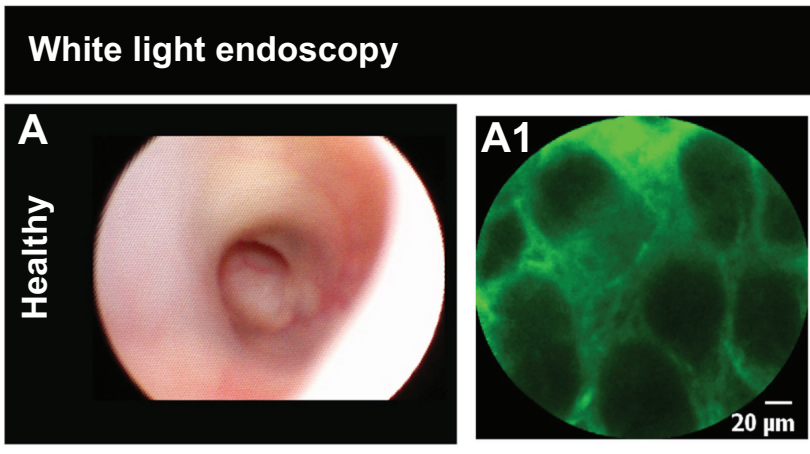

\section{Dual band CLE}
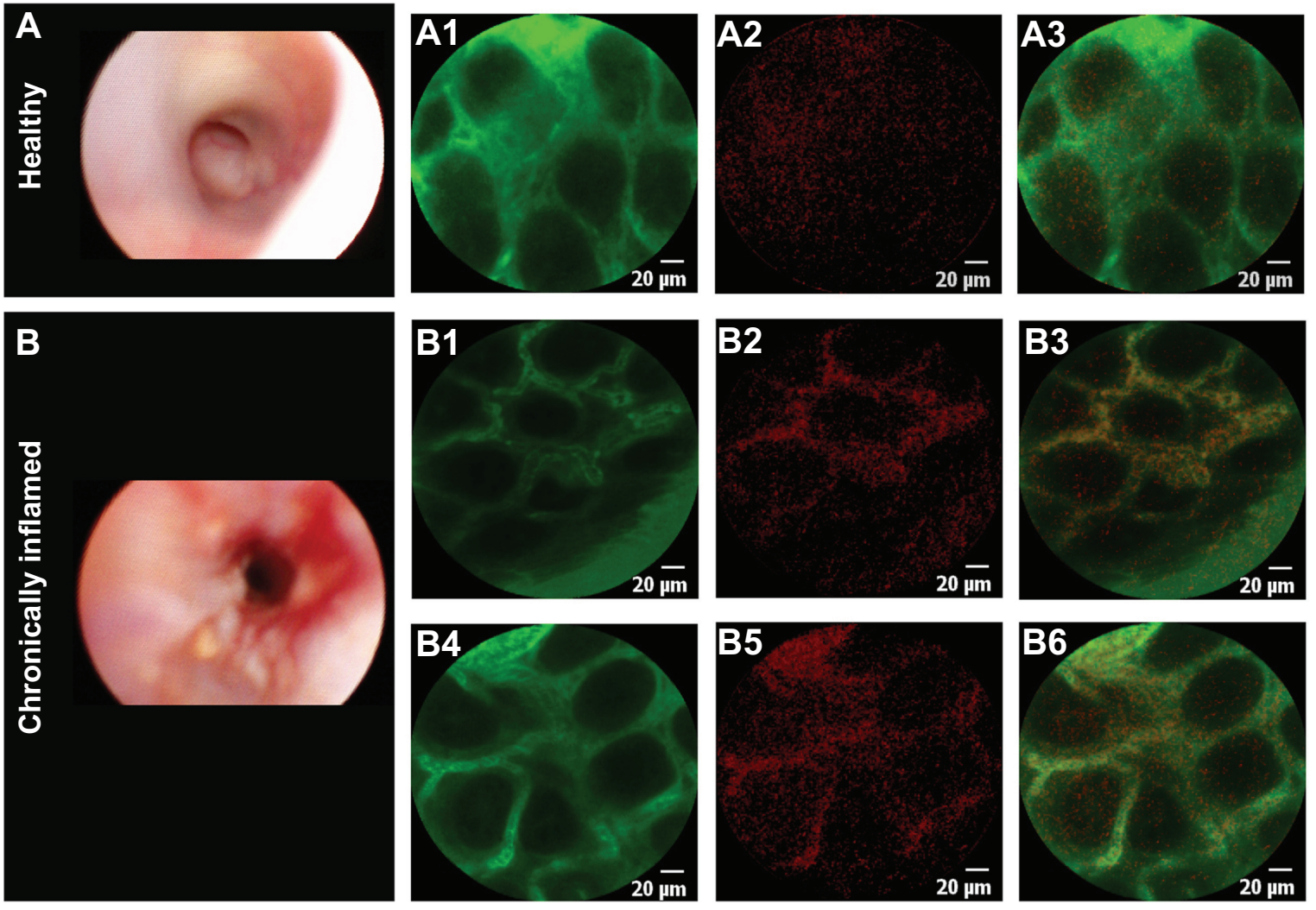

Figure 3 Differential nanoparticle distribution in inflamed and healthy mucosa.

Notes: Selected images of healthy $(\mathbf{A})(n=3)$ and chronically inflamed $(\mathbf{B})(n=3)$ mouse mucosa, upon intravenous administration of ANANAS-red ${ }^{681}$ in phosphate-buffered saline containing $1 \%$ fluorescein. CLE images were acquired in the green (AI, BI, B4) and red (A2, B2, B5) channels. Merged red and green images are shown in panels A3,

B3, and B6. The yellow color corresponds to the merged signal from green and red channels.

Abbreviations: ANANAS, avidin-nucleic acid nanoassembly; CLE, confocal laser endomicroscopy.

nanoparticle platform as a tool for targeted delivery of diagnostic agents. It should be noted that due to the high compositional versatility of the platform and its ease of formulation and tuning, part or all of the diagnostic elements could be substituted with therapeutic agents, making the nanosystem a potential carrier for targeted drug delivery to inflamed mucosa. The results are encouraging for future development of the platform, although additional studies are necessary before translation into clinical practice. On the diagnostic side, future experiments aimed at determining the duration of distribution and optimization of nanoparticle concentration are warranted. In the context of targeted drug delivery, research should focus on the development of linker technologies to enable efficient local release of the biotin-linked bioactive agent as soon as the nanoparticles reach their target.

\section{Acknowledgments}

This research was funded by the Roberto Farini Association, Progetti di Ateneo, and Ex 60\% at the University of Padova and National PRIN 2010-2011. We are grateful to ANANAS Nanotech for assistance with preparation and characterization of the nanoparticles, to Dr G Battaglia for promoting the acquisition and availability of the Mauna Kea system for CLE at the Venetian Institute of Molecular Medicine, Padova, and to Dr C Giacometti for advice on histology analysis.

\section{Disclosure}

$\mathrm{MM}$ is among the inventors of the ANANAS technology, and is a shareholder in ANANAS Nanotech s.r.l., a spinoff company that holds the intellectual property related to the ANANAS formulation. The other authors report no conflicts of interest in this work.

\section{References}

1. Gasparetto M, Guariso G. Highlights in IBD epidemiology and its natural history in the paediatric age. Gastroenterol Res Pract. 2013; 2013:829040.

2. Guariso G, Gasparetto M, Visona Dalla Pozza L, et al. Inflammatory bowel disease developing in paediatric and adult age. J Pediatr Gastroenterol Nutr. 2010;51(6):698-707. 
3. Hold GL, Smith M, Grange C, Watt ER, El-Omar EM, Mukhopadhya I. Role of the gut microbiota in inflammatory bowel disease pathogenesis: what have we learnt in the past 10 years? World J Gastroenterol. 2014;20(5):1192-1210.

4. Feagan BG, Yan S, Bala M, Bao W, Lichtenstein GR. The effects of infliximab maintenance therapy on health-related quality of life. Am J Gastroenterol. 2003;98(10):2232-2238.

5. Hanauer SB, Feagan BG, Lichtenstein GR, et al. Maintenance infliximab for Crohn's disease: the ACCENT I randomised trial. Lancet. 2002; 359(9317):1541-1549.

6. Lichtenstein GR, Yan S, Bala M, Hanauer S. Remission in patients with Crohn's disease is associated with improvement in employment and quality of life and a decrease in hospitalizations and surgeries. Am J Gastroenterol. 2004;99(1):91-96.

7. Sands BE, Blank MA, Patel K, van Deventer SJ. Long-term treatment of rectovaginal fistulas in Crohn's disease: response to infliximab in the ACCENT II Study. Clin Gastroenterol Hepatol. 2004; 2(10):912-920.

8. Noman M, Baert F, Get DH. HACA formation after infliximab (Remicade) treatment in Crohn's disease is clearly associated with infusion reactions. Gastroenterology. 2001;120(5 Suppl 1):A621.

9. European Medicines Agency. EPAR Remicade Summary of product characteristics. European Medicines Agency; 2009. Available from: http://www.ema.europa.eu/docs/en_GB/document_library/EPAR_Product_Information/human/000240/WC500050888.pdf. Accessed November 6, 2014.

10. De Rosa FG, Shaz D, Campagna AC, Dellaripa PE, Khettry U, Craven DE. Invasive pulmonary aspergillosis soon after therapy with infliximab, a tumor necrosis factor-alpha-neutralizing antibody: a possible healthcare-associated case? Infect Control Hosp Epidemiol. 2003;24(7):477-482.

11. Helbling D, Breitbach TH, Krause M. Disseminated cytomegalovirus infection in Crohn's disease following anti-tumour necrosis factor therapy. Eur J Gastroenterol Hepatol. 2002;14(12):1393-1395.

12. Sarzi-Puttini P, Ardizzone S, Manzionna G, et al. Infliximab-induced lupus in Crohn's disease: a case report. Dig Liver Dis. 2003;35(11):814-817.

13. Moghimi SM. Bionanotechnologies for treatment and diagnosis of Alzheimer's disease. Nanomedicine. 2011;7(5):515-518.

14. Matsumura Y, Maeda H. A new concept for macromolecular therapeutics in cancer chemotherapy: mechanism of tumoritropic accumulation of proteins and the antitumor agent SMANCS. Cancer Res. 1986;46(12 Pt 1):6387-6392.

15. Kiesslich R, Duckworth CA, Moussata D, et al. Local barrier dysfunction identified by confocal laser endomicroscopy predicts relapse in inflammatory bowel disease. Gut. 2012;61(8):1146-1153.

16. Wilchek M, Bayer EA. Introduction to avidin-biotin technology. Methods Enzymol. 1990;184:5-13.

17. Bigini P, Previdi S, Casarin E, et al. In vivo fate of avidin-nucleic acid nanoassemblies as multifunctional diagnostic tools. ACS Nano. 2014;8(1):175-187.
18. Shiekh FA. Targeted nanotherapeutics in cancer. Int J Nanomedicine. 2014;9:1627-1628.

19. Morpurgo M, Facchin S, Pignatto M, Silvestri D, Casarin E, Realdon N Characterization of multifunctional nanosystems based on the avidinnucleic acid interaction as signal enhancers in immuno-detection. Anal Chem. 2012;84(7):3433-3439.

20. Kiesslich R, Goetz M, Vieth M, Galle PR, Neurath MF. Technology insight: confocal laser endoscopy for in vivo diagnosis of colorectal cancer. Nat Clin Pract. 2007;4(8):480-490.

21. De Palma GD, Rispo A. Confocal laser endomicroscopy in inflammatory bowel diseases: dream or reality? World J Gastroenterol. 2013;19(34): 5593-5597.

22. Neumann H, Kiesslich R. Endomicroscopy and endocytoscopy in IBD. Gastrointest Endosc Clin N Am. 2013;23(3):695-705.

23. Buda A, Hatem G, Neumann H, et al. Confocal laser endomicroscopy for prediction of disease relapse in ulcerative colitis: a pilot study. J Crohn's Colitis. 2013;8(4):304-311.

24. Gomez V, Buchner AM, Dekker E, et al. Interobserver agreement and accuracy among international experts with probe-based confocal laser endomicroscopy in predicting colorectal neoplasia. Endoscopy. 2010;42(4):286-291.

25. Shahid MW, Buchner A, Gomez V, et al. Diagnostic accuracy of probe-based confocal laser endomicroscopy and narrow band imaging in detection of dysplasia in duodenal polyps. J Clin Gastroenterol. 2012;46(5):382-389.

26. Goyal N, Rana A, Ahlawat A, Bijjem KR, Kumar P. Animal models of inflammatory bowel disease: a review. Inflammopharmacology. 2014; 22(4):219-233.

27. Okayasu I, Hatakeyama S, Yamada M, Ohkusa T, Inagaki Y, Nakaya R. A novel method in the induction of reliable experimental acute and chronic ulcerative colitis in mice. Gastroenterology. 1990; 98(3):694-702.

28. Perse M, Cerar A. Dextran sodium sulphate colitis mouse model: traps and tricks. J Biomed Biotechnol. 2012;2012:718617.

29. Pignatto M, Realdon N, Morpurgo M. Optimized avidin nucleic acid nanoassemblies by a tailored PEGylation strategy and their application as molecular amplifiers in detection. Bioconjug Chem. 2010;21(7):1254-1263.

30. Morpurgo M, Radu A, Bayer EA, Wilchek M. DNA condensation by high-affinity interaction with avidin. Mol Recognit. 2004;17(6): 558-566.

31. Becker V, van den Broek FJ, Buchner AM, et al. Optimal fluorescein dose for intravenous application in miniprobe-based confocal laser scanning microscopy in pigs. J Biophotonics. 2010;4(1-2):108-113. 


\section{Supplementary material}

Figure $\mathrm{S} 1$ shows the gel permeation chromatography analysis of biotin- $\mathrm{C}_{6}-$ red $^{681}$ (compound 3 ) before and after addition of avidin. Figure $\mathrm{S} 2$ shows the fluorescence intensity calibration curves of the same compound in phosphatebuffered saline, alone and in the presence of avidin. Figure S3 shows the results of dynamic light scattering analysis of the avidin-nucleic acid nanoassembly (ANANAS)-red ${ }^{681}$ nanoparticles. Figure S4 shows histological images of colonic mucosa in dextran sodium sulfate (DSS)-treated and healthy mice.

\section{Characterization of biotin- $\mathrm{C}_{6}-$ red $^{681}$}

For analysis, a small aliquot of the coupling reaction solution $(0.5 \mu \mathrm{L})$ was diluted to $218 \mu \mathrm{L}$ with phosphate-buffered saline alone or phosphate-buffered saline containing avidin $1 \mathrm{mg} / \mathrm{mL}$ (Belovo Chemicals, Bastogne, Belgium), and analyzed by fast protein liquid chromatography (Superdex peptide column with phosphate-buffered saline as the eluent, $0.5 \mathrm{~mL} / \mathrm{min}$, ultraviolet absorption $681 \mathrm{~nm}$ ). Figure $\mathrm{S} 1$ shows the overlapped chromatograms of the starting red ${ }^{681}$ NHS, the reaction product as before and after the addition of avidin, with peak assignments. The hydrolyzed red ${ }^{681}$-NHS ( $\left.\operatorname{Red}^{681}-\mathrm{COOH}\right)$ and its biotin derivative were tested for fluorescence intensity. The biotin derivative was also tested upon addition of avidin. Fluorescence intensity calibration curves

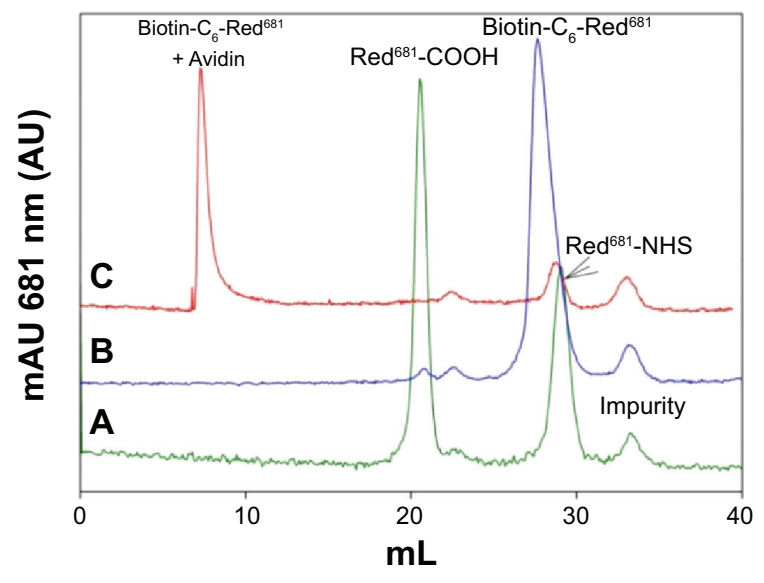

Figure SI Chromatographic analysis of red ${ }^{681}$ derivatives. (A) Starting NHS reagent, (B) biotin- $\mathrm{C}_{6}-$ red $^{681}$ derivative (compound 3), and (C) compound 3 in the presence of avidin.

Notes: Analyses were carried out using fast protein liquid chromatography apparatus (Akta Purifier, GE Healthcare, Little Chalfont, UK) equipped with a Superdex peptide column and eluted with $10 \mathrm{mM}$ phosphate, $150 \mathrm{mM} \mathrm{NaCl}, \mathrm{pH}$ 7.4 (phosphate-buffered saline) at $0.5 \mathrm{~mL} / \mathrm{min}$. Chromatograms were registered at $681 \mathrm{~nm}$.

Abbreviations: ANANAS, avidin-nucleic acid nanoassembly; NHS, $\mathrm{N}$-hydroxysuccinimide.

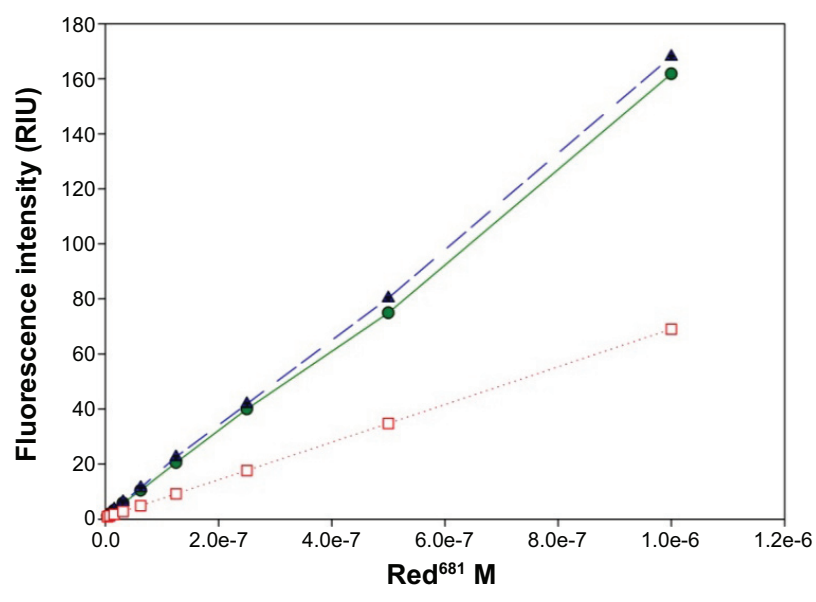

Figure S2 Fluorescence intensity versus concentration of $\operatorname{red}^{681}-\mathrm{COOH}(\bullet)$ and biotin- $\mathrm{C}_{6}$-red ${ }^{681}$ as before $(\boldsymbol{A})$, and in the presence of avidin $(\square)$.

Abbreviation: RIU, relative intensity units.

were generated on serially diluted phosphate-buffered saline solutions, starting from $1 \mu \mathrm{M}$ red ${ }^{681}$, with $\lambda_{\text {exc }} 681 \mathrm{~nm}$ and $\lambda_{\mathrm{em}} 708 \mathrm{~nm}$. The slope of the fluorescence intensity versus concentration curve was used to calculate the relative fluorescence intensity with respect to the starting $\mathrm{red}^{681}-\mathrm{COOH}$. The fluorescence intensity of avidin-linked biotin- $\mathrm{C}_{6}-\mathrm{red}^{681}$ was $43 \%$ that of the same reagent when analyzed free in solution. The same result was obtained using ANANAS instead of avidin (not shown).

\section{Size distribution of ANANAS nanoformulation}

Dynamic light scattering measurements were performed using a Malvern Zetasizer Nano ZS (Malvern Instruments Ltd, Malvern, UK) and the results are shown in Figure S3.

\section{Induction of DSS chronic colitis}

Chronic colitis was induced in Balb/c female mice ${ }^{1,2}$ (aged 8-10 weeks) by using multiple cycles of dextran sodium sulfate (DSS, $40 \mathrm{kDa}$, Sigma-Aldrich, St Louis, MO, USA). We performed preliminary optimization experiments to identify the optimal DSS dose and administration schedule yielding chronic inflammation. During treatment, animal body weight, rectal bleeding, stool consistency, and the presence of blood in stools were assessed daily. Assessment of chronic inflammation was confirmed by histological analysis. The final induction protocol identified is reported below: initially, we administered two cycles comprising 7 days of 3\% DSS in drinking water and 3 days of untreated drinking water. Next, $5 \%$ DSS was administered for 3-4 days until a $10 \%$ weight 
A Size distribution by intensity

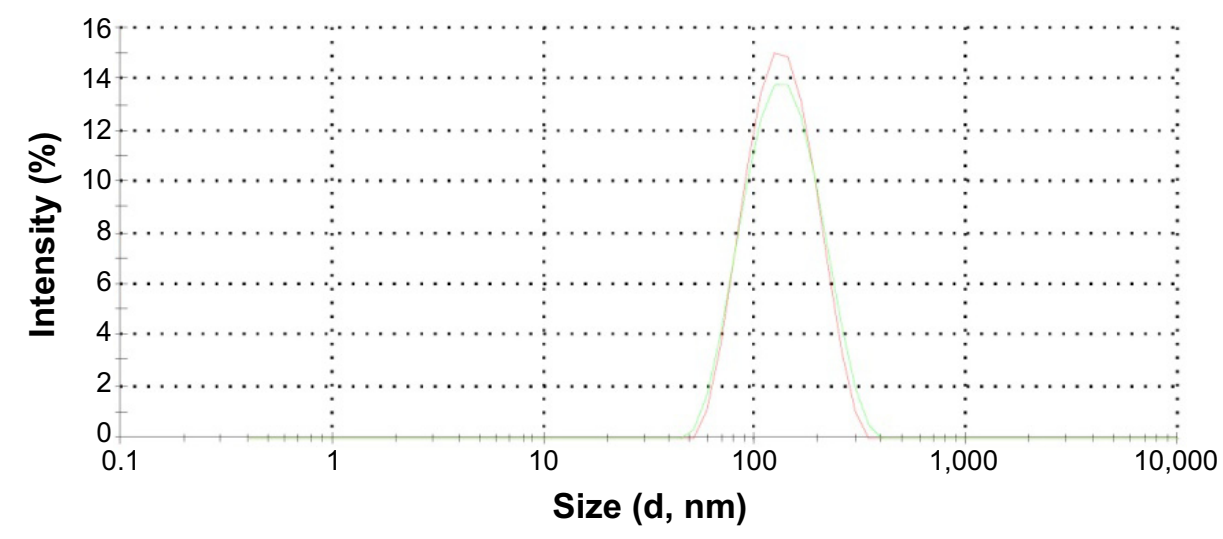

B Size distribution by volume

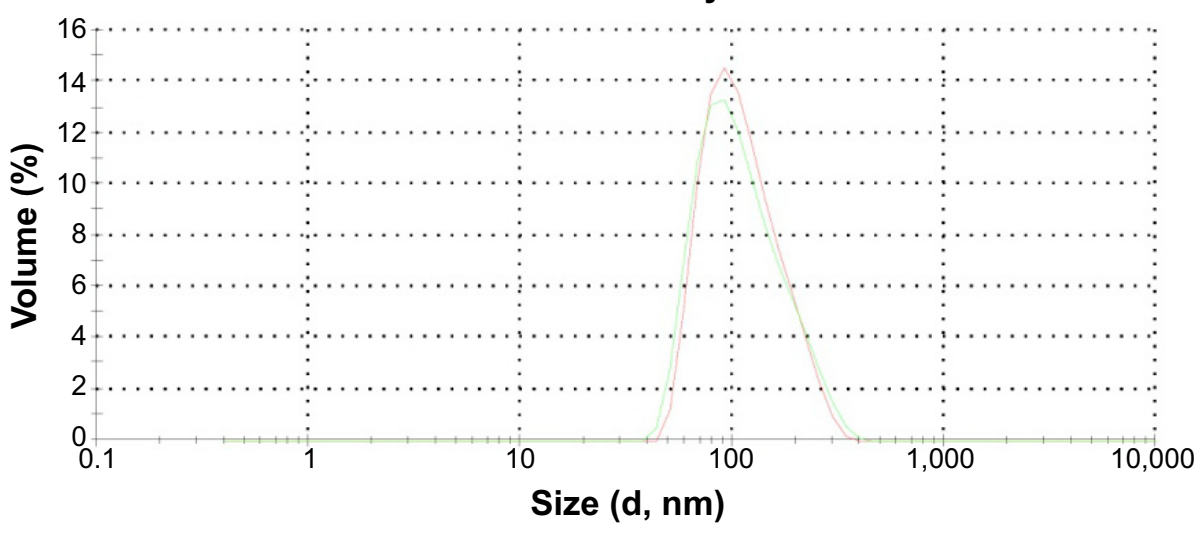

\begin{tabular}{|c|c|c|}
\hline \multicolumn{3}{|c|}{ ANANAS formulation size distribution (nm) } \\
\hline By intensity & By volume & Z-average \\
\hline $143 \pm 55$ & $118 \pm 57$ & 123.0 \\
\hline
\end{tabular}

Figure S3 (A) Intensity and (B) volume weighted size distribution of ANANAS assembly prepared for this work, as determined by dynamic light scattering. Abbreviation: ANANAS, avidin-nucleic acid nanoassembly.
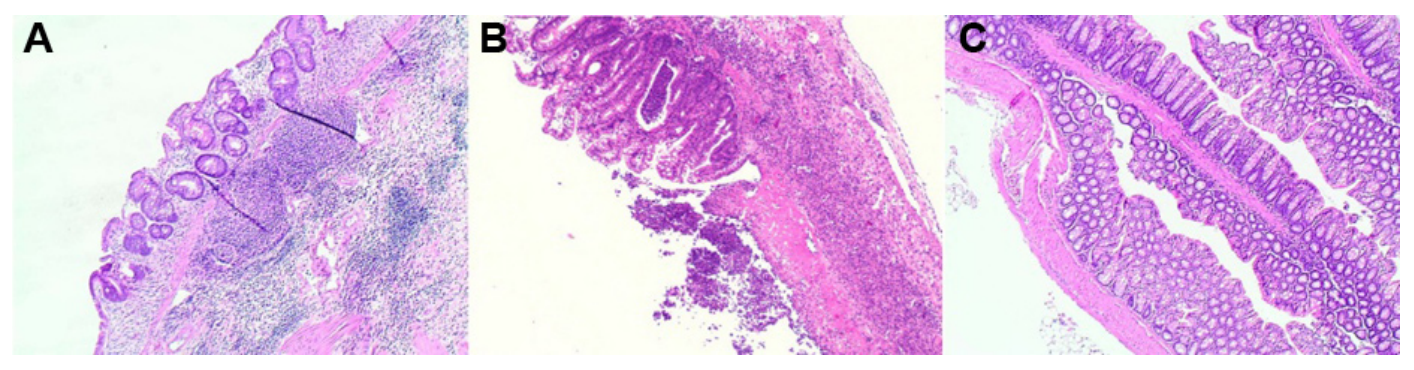

Figure S4 Histology images of dextran sodium sulfate-treated $(\mathbf{A}, \mathbf{B})$ and healthy $(\mathbf{C})$ mouse colonic mucosa. Notes: Colonic segments, fixed in $4 \%$ formaldehyde, were stained with hematoxylin and eosin and reviewed by a pathologist in a blinded fashion. Original magnification for histology, 40x.

loss was achieved, then substituted with untreated drinking water for 3 days or until full body weight recovery. The $5 \% \mathrm{DSS} /$ untreated drinking water cycle was repeated four times. Inflammatory changes ${ }^{3}$ (crypt architectural disarray, transmural inflammation with lymphoid follicles) were confirmed histologically in colonic sections from DSS-treated mice compared with control mice (Figure S4).

\section{References}

1. Hatakeyama S, Yamada M, Ohkusa T, Inagaki Y, Nakaya R. A novel method in the induction of reliable experimental acute and chronic ulcerative colitis in mice. Gastroenterology. 1990;98(3):694-702.

2. Goyal N, Rana A, Ahlawat A, Bijjem KR, Kumar P. Animal models of inflammatory bowel disease: a review. Inflammopharmacology. 2014;22(4):219-233.

3. Perse M, Cerar A. Dextran sodium sulphate colitis mouse model: traps and tricks. J Biomed Biotechnol. 2012;2012:718617. 


\section{Publish your work in this journal}

The International Journal of Nanomedicine is an international, peerreviewed journal focusing on the application of nanotechnology in diagnostics, therapeutics, and drug delivery systems throughout the biomedical field. This journal is indexed on PubMed Central,

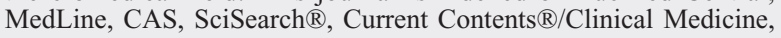

Journal Citation Reports/Science Edition, EMBase, Scopus and the Elsevier Bibliographic databases. The manuscript management system is completely online and includes a very quick and fair peer-review system, which is all easy to use. Visit http://www.dovepress.com/ testimonials.php to read real quotes from published authors.

Submit your manuscript here: http://www.dovepress.com/international-journal-of-nanomedicine-journal 